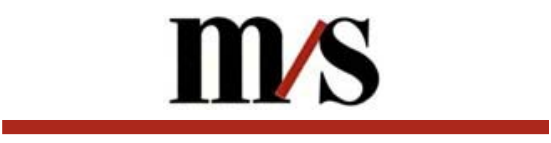

médecine/sciences $1995 ; 11: 315-6$

\title{
médecine/sciences A 10 ANS, LA MÉDECINE ET LA SCIENCE DANS DIX ANS
}

I l y a 12 ans, à l'initiative des gouvernements français et quebécois se réunissait de part et d'autre de l'Atlantique, un petit groupe de médecins et de biologistes, avec Jean Hamburger, dont le but était de lancer une revue francophone de qualité se consacrant à l'interface entre médecine et biologie. Il eut été alors probablement bien difficile de convaincre la majorité des auteurs actuels de médecine/sciences que se créait là, sous leurs yeux, une revue dans laquelle ils voudraient avec tant d'intensité s'exprimer, présentant leur démarche et leurs résultats dans une langue dont beaucoup d'entre eux avaient oublié qu'elle avait vocation à véhiculer l'information scientifique autant que d'autres aspects de la culture dans son sens le plus traditionnel. A dire vrai, l'équipe fondatrice de médecine/sciences, certes pleine d'énergie et de foi, ne pouvait elle-même imaginer que son enfant acquerrait un tel poids. Il en va de beaucoup d'entreprises comme de la création artistique et littéraire : l'oeuvre en vient à contraindre le créateur presqu'au-tant qu'elle en est l'accomplissement. Ainsi, médecine/ sciences avance-t-elle aujourd'hui mue par sa propre énergie, celle que lui insuffle une communauté scientifique d'autant plus exigeante qu'elle s'est laissée conquérir.

Outre une progression continue des abonnements à médecine/sciences, qui ne s'est jamais démentie depuis 10 ans (figure 1), plusieurs données témoignent de l'existence d'un véritable "phénomène médecine/sciences". Tout d'abord le nombre toujours croissant d'excellents manus- crits acceptés pour publication après une triple évaluation anonyme, manuscrits dont l'accumulation et, en conséquence, le retard à la publication constituent les seules difficultés réelles auxquelles médecine/sciences soit confrontée aujourd'hui. Puis, différents indices de l'internationalisation de notre audience. Parmi les quelques 1500 abonnements institutionnels à médecine/sciences, avant tout des bibliothèques, plus de 500 sont situés hors de France et du Québec. Mieux même, et le phénomène devient là particulièrement spectaculaire, ce sont les grandes communautés scientifiques francophones situées hors de France qui manifestent le désir de se présenter sous leur meilleur jour dans une revue de prestige et demandent de prendre en charge la préparation d'un numéro de médecine/sciences consacré à leur établissement. Après le laboratoire Européen de Biologie Moléculaire (EMBL) de Heidelberg, ce fut l'Université de Montréal, puis, en février 1995, l’Université Libre de Bruxelles. D'ici la fin de l'année, nos lecteurs pourront découvrir sous son meilleur jour la recherche biomédicale de l'Université Catholique de Louvain et des contacts ont été pris avec l'Université de Genève et une autre Université québécoise.

Dans le sillage du succès de médeci$n e /$ sciences, c'est à une véritable résurrection de la crédibilité de la publication biomédicale d'excellence en français que l'on a assisté, avec le lancement ou la transformation de nombreuses revues. Cependant, que médecine/sciences soit rédigée en français n'explique évidemment pas son suc-
A. Kahn.

$m / s n^{\circ} 3$, vol. 11, mars 95 
cès, même si cela lui donne une signification particulière. médecine/ sciences est née alors que l'on commençait à entrevoir l'application à la médecine des retombées de la révolution du génie génétique, apparu 10 ans auparavant, a u début de l'épidémie du SIDA et au cœur de l'explosion des méthodes informatisées de traitement des signaux et des données, et donc de la transformation des techniques d'imagerie. Les grandes découvertes des dernières décennies en matière thérapeutique avaient été faites, transformant le traitement et le pronostic de maladies fréquentes telles que l'hypertension artérielle ou l'ulcère gastro-duodénal. La ciclosporine était le chef de file d'une nouvelle famille d'immunosuppresseurs améliorant le résultat des greffes d'organes et, donc, en élargissant les indications. Cependant, peu de progrès étaient faits dans le domaine des maladies neurologiques, des maladies dégénératives en général, du cancer, des maladies génétiques; de nouvelles souches, voire de nouvelles espèces d'agents infectieux étaient caractérisées. Pour l'essentiel, il semblait que les méthodes habituelles d'accès aux nouveaux traitements, de création de nouveaux médicaments devenaient peu opérationnelles, et qu'il fallait donc, pour améliorer la prise en charge thérapeutique d'affections visà-vis desquelles la médecine était insuffisamment efficace, faire appel à de nouvelles stratégies fondées plus sur la conception rationnelle à partir de données physiopathologiques et moléculaires que sur le criblage semialéatoire de molécules nombreuses. Le génie génétique et ses développements en génétique humaine et biologie moléculaire devaient donner accès aux déterminants des maladies, et donc aux cibles des médicaments de demain. Tel est l'un des postulats fondateurs de médecine/sciences, justifiant ce que fut la revue ces dix dernières années. Peut-être, l'une des raisons principales de notre succès est-elle que cette analyse est aujourd'hui généralement acceptée, et que nous en avons été d'efficaces artisans. Que cette lucidité de médeci- moins insuffisant à expliquer la place qu'elle a prise dans notre communauté de biologistes et de médecins ; il fallait aussi qu'elle fût servie par ce qui est incontestablement notre originalité, la mobilisation pour et autour d'elle d'un nombre croissant des meilleurs chercheurs de nos domaines, acceptant non seulement de présenter une synthèse des connaissances, mais aussi d'en faire chaque fois une analyse critique, d'oser des interprétations originales, des pronostics et des hypothèses. Certes, on trouve un peu l'équivalent dans ces glorieuses revues généralistes que sont Nature et Science, mais pas au même niveau : médecine/ sciences, ce sont dans chaque numéro, une dizaine de mini-synthèses et de nouvelles, des dizaines de nouvelles brèves, toutes écrites par d'authentiques et reconnus spécialistes des sujets présentés ; ils étaient 161, rien qu'en 1994, à avoir contribué à ce type de diffusion de l'information scientifique. Les interrogations, sur les dimensions épistémologique sociale, économique et éthique des techniques nouvelles dérivées de tant de connaissances récemment acquises sont évidemment celles de beaucoup d'entre nous, et ont donc tout naturellement trouvé, elles aussi, un écho dans notre revue, sous la forme de rubriques spéciales.

Ce numéro anniversaire a été conçu comme une illustration du style méde cine/sciences: certains des scientifiques les plus actifs en biologie et en médecine de ces dernières années tentent d'éclairer l'avenir à la lumière des progrès récemment réalisés : les programmes Génome, servis par le développement de l'informatique et de la robotique, débouchent sur la découverte des gènes de maladies génétiques, de cancers et de cibles diverses de médicaments futurs. Le concept d'apoptose réunit aujourd'hui des phénomènes essentiels du développement, de la réponse immunitaire, de la cancérisation, de la sénescence et de nombreux syndromes dégénératifs. La décennie passée est aussi celle de l'explosion de nos connaissances sur la nature et la transmission des signaux entre les cellules et au sein de chacune d'entre elles, relayée par le langage moléculaire des cytokines et les cascades de phosphorylations et déphosphorylations aboutissant à la régulation de tous les aspects de la vie cellulaire : cycle cellulaire, transcription, métabolisme, sécrétion, etc. Malgré la diversité des outils du génie génétique et de la biologie moléculaire, les progrès décisifs en matière de traitement du SIDA tardent, la masse des connaissances virologiques et immunologiques accumulées sur le VIH expliquant les difficultés rencontrées et engendrant continuellement de nouvelles perspectives qu'il vaut la peine d'explorer. La maîtrise acquise de la fécondité féminine, au moins dans les pays industrialisés, a rendu paradoxalement insupportable l'infécondité, même toute relative, stimulant le développement de diverses méthodes d'assistance médicale à la procréation de plus en plus interventionnistes.

De la procréation à la sénescence et à la mort, en passant par la lecture du programme génétique et les recherches sur les grandes maladies d'avenir, car aujourd'hui non maîtrisées (maladies neurologiques et dégénératives, désordres nutritionnels et diabète, maladies infectieuses et parasitaires, cancer, affections génétiques), ce numéro anniversaire de médecine/ sciences offre à nos lecteurs une vue circulaire telle qu'on peut l'avoir d'un lieu éminent (notre revue !) en 1995. Plus avant dans cette fin de siècle (et même de millénaire), nous explorerons plus en détail chacune de ces directions, la disponibilité et la motivation de nos lecteurs à nous aider nous assurant que nous n'oublierons aucune de celle dont notre avenir, scientifique et médical sera fait, et que nous n'en négligerons pas non plus les dimensions philosophique, sociale et morale. Notre prochain rendezvous dans des circonstances similaires va de soi : au XXI' siècle

Avec vous, lecteurs, grâce à vous, longue vie à médecine/sciences; façonnons-la à l'image du meilleur de nous-mêmes, afin qu'elle soit ainsi le reflet de ce que nous, biologistes et médecins francophones, voulons être et voulons dire 